\title{
Vitiligo - from clinical manifestations to pathophysiological mechanisms and cell death
}

\author{
Ana Maria Chivu', Elena Balasescu', Roxana Ioana Nedelcu',2, Alice Brinzea ${ }^{1,2}$, \\ Mihaela Antohe ${ }^{1,2}$, Anastasia Coman ${ }^{1,2}$, Gabriela Turcu ${ }^{1,2,3}$, Ionela Hulea', \\ Daniela Adriana Ion ${ }^{1}$ \\ 1"Carol Davila” University of Medicine and Pharmacy, Bucharest, Romania \\ ${ }^{2}$ Dermatology, Derma360 Clinic, Bucharest, Romania \\ ${ }^{3}$ Dermatology Department, Colentina Clinical Hospital, Bucharest, Romania
}

\begin{abstract}
The pathogenesis of vitiligo is still unclear. The complexity of the disease seems to be based on the intervention of environmental factors in the background of genetic predisposition which will determine the response of the immune system, the involvement of the nervous and endocrine systems, altered metabolisms and increased oxidative stress - all these elements leading to melanocytic dysfunction and their subsequent destruction. Melanocytic death has a clinical correspondent that embraces the form of white macules on the skin, associated or not with leucotrichia. A better understanding of vitiligo' s pathophysiology and mechanisms of cell death at the skin level can be a step to some appropriate treatment strategies.
\end{abstract}

Keywords: vitiligo, melanocytic dysfunction, pathophysiological mechanisms, cell death

\section{INTRODUCTION}

A cutaneous progressive disorder of pigmentation, vitiligo is characterized by whitish macules due to a progressive destruction of the melanocytes. The immune system, the endocrine and nervous systems intertwine in the form of "nodes" of vitiligo's pathogenesis. In the recent years, several "nodes" have managed to be untied, bringing the whole process of research closer to a "straight line" which could indicate in the future the path to a targeted, specifically and personalized therapy, and to prevent interventions on both environmental and genetic factors to avoid the onset of vitiligo. Till then, the proper diagnosis and classification in a specific category of vitiligo (1) and specific therapeutic indications are required in an attempt to slow its progression.

\section{CLINICAL TYPES}

Various skin areas may be affected, with a wide variability both in terms of lesion dimensions and their extent relative to the body surface. The main forms of vitiligo (Figure 1) are segmental (SV), non-segmental (NSV) and mixed vitiligo (2). Within the NSV category, the forms of generalized vitiligo $(\mathrm{GV})$ come in forms like: acro-facial, which affects the distal extremities and the face, at the level of which there are involved periocular and perioral areas, vulgar, in which Koebner phenomenon is highly expressed, implying areas of high pressure upon the skin and repeated micro-trauma, and the universal one, in which majority of the skin surface is affected by vitiligo, showing a white-patched macular pattern. Another clinical category of vitiligo, the segmental one, follows a dermatomer pattern (frequently the mandibular branch of the trigeminal nerve).

Segmental vitiligo represents approximately $10 \%$ of the total cases of vitiligo; it has an unilateral distribution, totally or partially affecting a well-defined segment (3). They may be interested in ipsi or contralateral dermatome depigmentation. The cephalic extremity is involved in over $50 \%$ of cases. The most frequently involved dermatome is 


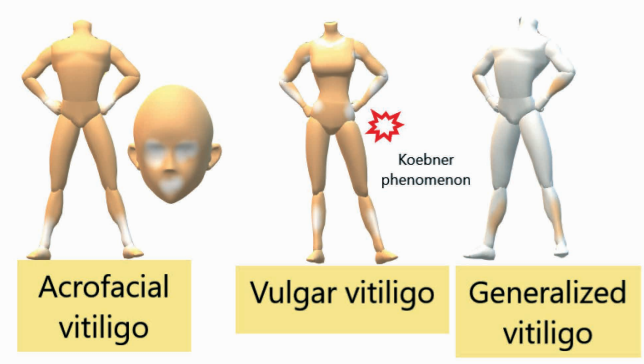

that of the trigeminal nerve (4). From an evolutionary point of view, it is associated with a rapid progression during the first two years from the onset and with the early appearance of leukotrichia. Leukotrichia occurs in approximately $8.9-45 \%$ of vitiligo cases and is a subsequent impairment of leukoderma, being also a negative prognostic factor and more common in segmental vitiligo (2). From a pathophysiological perspective, this form of vitiligo involves the dysfunction of regulatory $\mathrm{T}$ lymphocytes, through a decrease in immunological tolerance to the population of stem cells in the hair follicle (5). These forms associated with leukotrichia are highly resistant to treatment (6), therefore the mesenchymal stem cell reservoir is absent from the hair follicle and cannot be stimulated during the therapy.

Non-segmental vitiligo often has bilateral and symmetrical distribution. Named also generalized vitiligo, it is the most common form of vitiligo and mainly affects areas that tend to be exposed to pressure or micro trauma (4). Two phenotypic forms of VNS have been differentiated: the early onset form (before the age of 12 years), frequently associated with halo nevi and a family history of premature hair graying and a form with an acro-facial distribution and late onset (4).

The form of acro-facial vitiligo affects the extremities, by the presence of depigmented spots on the hands and feet, with distal depigmentation of the fingers (4), accompanied by the presence of lesions on the face and neck. The facial orifices are mainly interested; it was described a subdivision the "liptype" shape that affects only the skin area of the lips and fingertips. Acro-facial vitiligo is a more common form among the South Asia's population and is generally a treatment-resistant form (6).

Vitiligo vulgaris, the most common form of vitiligo, symmetrically affects multiple body areas; Koebner phenomenon (characterized by the appearance of new depigmentation in the skin subjected to trauma) is highly expressed. Examples of these areas are the pelvic griddle, following mi-
FIGURE 1. Clinical forms of vitiligo - graphic representation in Paint $3 D$ by Ana Maria Chivu cro-trauma caused by wearing belts, or neck area, following the pattern of necklaces.

Forms of universal vitiligo are generally evolutionary forms affecting almost the entire skin area (6) and can also be accompanied by depigmentation of the mucous membranes and hair.

Recently have been described hypochromic or minor vitiligo (observed in patients with dark skin, and partial facial and torso depigmentation) and follicular vitiligo (which involves depigmentation of the hair without affecting the surrounding skin area, at least initially) (7). Follicular vitiligo has a distinct pathogenesis, primarily reducing the melanocytic reserve of the hair follicle, with the consequent depigmentation without affecting the melanocytes at the inter-follicular level. Frequently, the scalp hair and eyelashes are interested (2).

In rare cases, patients may have a specific impairment of segmental vitiligo forms concomitant with lesions developed outside depigmentation, this being framed in mixed vitiligo forms. Patients with mixed vitiligo benefit from a good response to phototherapy of the areas that do not affect the skin according to a segmental pattern, while segmental vitiligo lesions are generally poorly responsive (6). This low-frequency form among the affected population is characterized by an onset that includes segmental vitiligo treats, followed by bilateral impairment with variable temporal progression (which takes usually several months) (7).

Another rare subtype of vitiligo is mucosal, which involves restricted damage to the oral or genital mucosa (2).

Focal vitiligo involves the interest without a specific pattern of distribution of specific skin areas, small (10-15 $\left.\mathrm{cm}^{2}(6)\right)$, isolated and which has not evolved for a period of 1-2 years. It can later evolve into both VS and VNS (4).

Dotted vitiligo involves damage by dotted spots that may affect any skin segment. The macules have dimensions between 1-1.5 $\mathrm{mm}$ and if they do not coexist with classical vitiligo macules, they should be classified as "dotted leukoderma" (4). 
Vitiligo trichrome is characterized by the presence of a hypo-pigmented area between the depigmented area and the surrounding intact skin. This pattern of distribution suggests slow and centrifugal progression compared to other forms of vitiligo (8). This form of presentation is often associated with an active, progressive form of the disease (6), being one of the clinical markers that reveals the activity, along with the Koebner phenomenon and the confetti depigmentation pattern (6). The confetti depigmentation pattern is clinically characterized by numerous depigmented macules between $1 \mathrm{~mm}$ and $5 \mathrm{~mm}$, grouped generally at the edge of the extensively affected areas (6).

Inflammatory vitiligo is noted by highlighting an inflammatory, erythematous margin that delimits the lesion from the surrounding epidermis (8), skin biopsies highlighting the presence of a lymphocytic and histiocytic infiltrate at this level.

\section{EPIDEMIOLOGY}

Vitiligo is the most common acquired leukoderma (2), affecting $0.5-2 \%$ of worldwide population (4). $15-20 \%$ of patients have a first-degree relative with the same pathology and, in case of family aggregation, the transmission is non-Mendelian (7). A family history of vitiligo is associated with the early onset of the disease. The genetic component plays an important role in the pathophysiology of this disease. The genes identified as being involved are responsible for processes such as melanogenesis, immune tolerance and cellular apoptosis (4).

The condition is found in both sexes, without a specific predominance and can affect any race. The involvement of tyrosinase, the enzyme with a role in the process of melanin biosynthesis has been demonstrated (9), catalyzing the hydroxylation of tyrosine and its consequent oxidation (10). Tyrosinase has a distinct interracial activity, more intense in the melanocytes of the African-American population, than in the case of the Caucasian population (8).

In terms of the age of onset, a higher incidence was observed around the age of 20, and the age segment between 10-30 years old comprises most cases of vitiligo (4). The earliest reported case was immediately after birth, while the latest onset was observed in a 54-year-old patient (4). Segmental vitiligo tends to appear earlier in life. Over $90 \%$ of patients with vitiligo have the vulgar or acro-facial form as forms of generalized vitiligo. The other percentages are divided between localized vitiligo, more common than mixed or universal forms. At the same time, segmental vitiligo is more common among the pediatric population and the incidence of the association of endocrine pathologies is lower than in the case of the adult population.

\section{PATHOGENESIS}

Vitiligo is a multifactorial condition that involves the loss of functional melanocytes. Several pathophysiological mechanisms have been proposed: an autoimmune response against functional melanocytes, oxidative stress, detachment of melanocytes and the generation of inflammatory mediators (4). These hypotheses are not mutually exclusive, but could act synergistically - specific depigmentation can occur in the context of various melanocyte destruction mechanisms or can also be the result of a low renewal capacity of mesenchymal cells (11).

The autoimmunity hypothesis supports the existence of an immune-mediated substrate of the cellular apoptosis mechanism, involving both cytotoxic $\mathrm{T}$ lymphocytes and autoantibodies directed against surface melanocyte antigens, in the context of an altered immune tolerance $(8,9,12)$. The involvement of autoimmunity in pathogenesis is supported by the presence of circulating antibodies against melanocyte proteins (13). As an expression of the involvement of cellular immunity in pathogenesis are the presence of activated CD $8+1 \mathrm{ym}-$ phocytes at the perilesional level. The number of CD8 + T lymphocytes appears to correlate with disease activity (9). In addition, the non-segmental form of vitiligo is more frequently associated with other autoimmune pathologies (Hashimoto's thyroiditis, Graves' disease, Addison's disease, type 1 Diabetes mellitus, psoriasis, inflammatory bowel disease, autoimmune poly-endocrine syndrome) (8). Melanocytes are more sensitive to immune-mediated or toxic-mediated attack compared to keratinocytes and fibroblasts, thus explaining the increased susceptibility to destruction by specific antibodies produced in vitiligo $(13,14)$.

Biochemical theory suggests the effect of the accumulation of reactive oxygen species in the skin, which leads to the activation of melanocyte metabolites involved in a process of auto-toxicity that results in melanocytic apoptosis $(4,12)$. Melanogenesis generates a pro-oxidant state in the skin (4) and in vitiligo, melanocytes are more susceptible to oxidative stress than normal. The pathophysiological events involved in the process of melanocyte destruction have the following sequence: the initial element incriminated is the mel- 
anocyte response to stress that determines the production of reactive oxygen species, which will successively alter the activity of the antioxidant system. The balance between antioxidant mechanisms and the production of oxidative stress markers is affected in favor of superoxide dismutase, malonyl aldehyde and reactive oxygen species. It is also involved in a dysfunction of antioxidant mechanisms, in which enzymes such as catalase, glutathione peroxidase, glutathione reductase, thioredoxin oxidase, superoxide dismutase intervene, whose activity is low, both at the skin and systemic level (4). In the "epidermal melanin unit", keratinocytes and melanocytes are interconnected both with each other and with the basement membrane by adhesion molecules, such as e-cadherins, b-cadherins and integrins (4). It has been observed that, in the presence of an altered redox status in the epidermis of patients with vitiligo, keratinocytes not only decrease numerically, but also appear to suffer from vacuolar degeneration resulting in apoptosis. Thus, the inability of keratinocytes to produce adequate amounts of melanocyte growth factors, such as stem cell factor (SCF), also results in melanocyte apoptosis. Another way in which oxidative stress can induce melanocytic apoptosis is through mitochondrial mechanisms, by releasing caspase-activating cytochrome $\mathrm{c}$ (this mechanism causes an imbalance between Bax levels, as a pro-apoptotic protein, and Bcl-2, as an anti-apoptotic protein). Abnormal morphological changes were also observed in the keratinocytes and melanocytes of patients with vitiligo, in the form of dilatation of the endoplasmic reticulum, vacuolar degeneration, heterochromatic nuclei, all these features converging to cellular apoptosis (15).

Other theories claim that intrinsic melanocyte defects make them more susceptible to many stressors, a mechanism mediated by cytotoxicity or a neural mechanism (16). Internal or external lesion stimuli triggers an inflammatory mechanism, in which proinflammatory cytokines are initially released along with neuropeptides produced by nerve endings, followed by vasodilation and a consecutive immune response (4).

It has been shown that some phenolic and catecholaminergic compounds have a structure similar to tyrosine, the tyrosinase substrate, which initiates melanin biosynthesis, and due to this structural similarity, the compounds may interfere with the physiological process of melanin synthesis, through competitiveness $(4,15,17,18)$. Neural hypothesis emphasizes the neuron-like functions of melanocytes (19) and highlights the negative effect of a neurochemical mediator that causes melanocyte destruction or inhibits melanin production. This hypothesis proposes the explanation of melanocytic apoptosis by the presence of an altered reaction of pigment cells derived from the neural crest in response to exposure to neuropeptides, catecholamines or catecholamine metabolites, in association with a generalized increase in the sympathoadrenal system $(18,19)$. The release of neuropeptides from the peripheral nerve contributes to melanocyte damage through a synergistic mechanism, intricate with the action of proinflammatory cytokines (15). In particular, the release of acetylcholine and norepinephrine from the cutaneous axonal terminals has been shown to be toxic to melanocytes. At the same time, corticotropin-releasing hormone $(\mathrm{CRH})$ secreted under stress activates skin mastocytes that will produce proinflammatory cytokines (17). The neural mechanism hypothesis was generated by the presence of the unilateral pattern of distribution in SV forms, the symmetrical, bilateral distribution of the lesions in NSV forms, the loss of pigmentation in areas with transverse myelitis or diabetic neuropathy (19). An argument in favor of this theory was the observation of the role of psychological stress as an aggravating and precipitating factor among patients with vitiligo.

It also was observed that in vitiligo, an inherited intrinsic defect of melanocytes prevents them from differentiating and growing. Depending on the course of the disease, melanocytes may be affected to a different degree in the same patient (7).

According to the "convergence theory" vitiligo arises through a combination of these pathogenic mechanisms (19).

\section{MECHANISMS OF MELANOCYTE DYSFUNCTION - THE ROLE OF CELL DEATH}

In the epidermal-melanin unit melanocytes and keratinocytes are responsible to producing and distribution of melanin, synthesized in melanosomes and translocated into keratinocytes via dendrites (20). Transformation of tyrosine into melanin and melanin distribution are energy consuming processes (14) and result in accumulation of reactive oxygen species (21). The melanocyte alteration activates $\mathrm{T}$ cells, responsible for triggering the inflammatory process, initiation and progression of disease (14).

Genetic predisposition and some environmental factors (repeated exposure to ultraviolet radia- 
tion, micro-trauma or the interaction with certain irritating chemicals) lead to high amounts of free radicals and hydrogen peroxide, with a consecutive activation of the immune system and a cytotoxic response directed against some melanocytes (22) that have an increased susceptibility to oxidative stress and a lower cell adhesion compared to normal melanocytes (Figure 2). Reactive oxygen species accumulation, inhibition of melano-genesis, dysregulation of protein expression, activation of unfolded protein response and initiation of autophagy are steps to melanocyte loss (23). This cascade of events will lead to melanocyte death, followed by skin depigmentation and leucotrichia.

The reduced redox status at the epidermal level leads to keratinocyte damage both numerically and morphologically, by vacuolation. In both melanocytes and keratinocytes were observed morphological changes such as dilations of the endoplasmic reticulum, vacuolar degeneration and the presence of heterochromatic nuclei, arguments for subsequent apoptotic cell death (15). Consecutively, keratinocyte production of Stem Cell Factor (SCF) decreases. Stem cell factor is an essential element for the survival of melanocytes (12), regulating both their growth and survival and thus, keratinocytes will promote melanocytic apoptosis (24). There are two isoforms of SCF: a fat-soluble, membrane-bound isoform ( $\mathrm{mSCF}$ ) and a water-soluble cytoplasmic isoform (cSCF). The membrane isoform serves to survive the melanocyte precursors at the dermal level, while the cytoplasmic form is necessary for the initial lateral dispersion of the melanocyte line and the subsequent survival at the site of intra-epidermal migration (24). Lowering adhesion to collagen type IV of melanocytes is an adhesion defect observed in vitiligo and support the theory of melanocytorrhagy as the primary defect underlying melanocyte loss in unstable vitiligo (16). Melanocytes migration to the supra-basal layers, to the spino cellular, granu- lar layer, and to the horny layer cause depigmentation through an isomorphic response (the Koebner phenomenon). In these atypical locations, melanocytes have a globular shape and don't present dendrites (25). Furthermore, SCF deficiency leads to cellular apoptosis (24). The most documented way of cell death in the pathogenesis of vitiligo remains apoptosis and only a minority of the melanocyte population suffers from the process of necrosis (11).

Cellular apoptosis in keratinocytes and melanocytes is controlled by the balance between anti-apoptotic and pro-apoptotic factors. Normal morpho-functional melanocytes express high levels of anti-apoptotic proteins (Bcl-2, Bcl-XL). Bcl-2 expression is closely correlated with caspase activation. Bcl-2 overexpression may inhibit caspase activation, while Bax overexpression may activate caspases (24).

The accumulation of oxygen radicals and low anti-oxidant cell capacity contributes to the alteration of cellular structures (26). Oxidative stress induces by mitochondrial mechanisms the release of cytochrome c activating caspase. The overproduction of free radicals at the epidermal level activates the pathway of pro-apoptotic Bax proteins which, from the cytosolic level after the activation, migrate to the mitochondrial surface, leading to the formation of transmembrane pores (Permeability Transition Pore or PT pore) (12). Following the formation of these mitochondrial pores, the mitochondrial activity decreases and is promoted the release of pro-apoptotic proteins, such as cytochrome c. From the moment of release of these proteins at cytosolic level, the activation of caspase- 9 is promoted and consecutively are activated other caspases (caspase-3, caspase-6) with alterations in cytoskeletal proteins (12). In this way, apoptosis of melanocytes is favored. In an in vitro study of melanocytes from non-lesional vitiligo skin were argued aberrant signal transduction pathways with




hyper-activation of mitogen-activated protein kinase, the $\mathrm{p} 53$ protein activation (25), morphological changes, cell hypertrophy, DNA alteration and premature onset of cell death $(21,26)$.

Cellular apoptosis involves two distinct main pathways: that of perforins or granzymes and the interaction between members of the tumor necrosis factor (TNF) family, Fas and its ligand, FasL (27). Perforins are pore-forming proteins found in the intracytoplasmic granules of cytotoxic $\mathrm{T}$ lymphocytes, which can lead to transient pore formation in the cell membranes of target cells. Among patients with vitiligo were observed higher levels of FasL and more important expression of perforins at the epidermal and dermal level compared to the cases in the control group (27). Moreover, the degree of perilesional infiltrate seems to correlate directly with the degree of activity and severity of the disease (27).

The reactive oxygen species produced in excess trigger the activation of cellular response to the unfolded protein with the melanocyte secretion of exosomes with melanocyte-specific antigens, microRNAs, heat shock proteins (Hsp) and damage-associated molecular patterns (DAMPs) (14). Therefore, through exosomes, specific antigens are brought into contact with the surrounding dendritic cells, which will further give rise to an immune response directed against these antigens.

Consequently, $\mathrm{T}$ helper lymphocytes are activated and occurs the $\mathrm{T}$ regulatory lymphocyte dysfunction, both processes being guided by specific cytokines and chemokines (14). The keratinocytes also synthesize pro-inflammatory cytokines, interleukin-6 and interleukin- 8 having a role in attracting T-cells to depigmented skin lesions (28). Cytokines, by binding to their specific receptor, determine the activation of the Janus kinase/signal transducers and activators of transcription (JAKSTAT) intracellular pathway $(29,30)$ through processes of auto-phosphorylation, dimerization, translocation at the nuclear level with consecutive binding to DNA and regulation of gene expression.

At patients with vitiligo, it has been observed that regulatory $\mathrm{T}$ lymphocytes (which play an essential role in immune tolerance) are dysfunctional and less numerical (31). In this context, the transcription factor responsible for down-regulating $\mathrm{T}$ lymphocyte activation, FoxP3, is significantly reduced (32).

Interferon gamma (essential in the recruitment of melanocyte-specific autoreactive $\mathrm{T}$ lymphocytes) inhibits melanogenesis and directly causes melanocyte apoptosis (4). Interferon gamma induces the production of chemokines, such as C-X-C motif chemokine ligand 10 (CXCL10) and the chemokine receptor CXCR3 (9), expressed in
TCD8+ lymphocytes, both locally - in the lesion, and also in the serum. This receptor and chemokine induction appears to be essential in the recruitment of T lymphocytes in the pathogenesis of vitiligo (6). Necrotic keratinocytes which release RNA are triggers for NF-kB activation, upregulation or intercellular adhesion melocule-1 (ICAM1) (33) and pro-inflammatory cytokine and chemokine secretion (9).

In abnormal conditions, melanocytes express ligands that can interact with the Natural Killer Group 2D (NKG2D) - a type II trans-membrane receptor expressed by CD8 T cells (26), facilitating the activation of CD8 + lymphocytes (34). NKG2D receptor is a recognition receptor of transformed cells involved in their elimination and can interact with a wide variety of ligands that are present in the context of cellular stress (26). The ligand-receptor interaction normally aims to destroy cancerous, infected or dysfunctional cells but has been shown to be closely related to NKG2D receptor expression in LTCD8 + and multiple autoimmune pathologies. In the skin areas affected by vitiligo were identified LTCD8 + expressing NKG2D receptor (34).

Resident CD8 + skin lymphocytes recognize melanocytes and destroy them $(31,35)$ through NKG2D and consequently LTCD $8+$ cell lines will develop, which, in the long run, will potentiate the production of pro-inflammatory cytokines and chemotactic agents that will amplify the whole process through a positive feedback loop, because this process will stimulate the expression in the cell membrane of NKG2D receptors and the recruitment of new $\mathrm{T}$ lymphocytes. This pathological vicious circle supports the perpetuation of the processes involved in the appearance and spread of the disease (34). Engagement of NKG2D receptor and IL-15 cytokine effects promote the memory formation and contribute at the cytotoxic effect on melanocyte of the tissue resident memory $\mathrm{T}$ cells population (26).

In vitiligo all these changes in melanocytes inter and intracellular systems are responsible for triggering the inflammatory process that underlies the onset and progression of the disease.

\section{CONCLUSIONS}

In the context of a genetic background, external or internal factors (such as trauma, ultraviolet radiation, phenols, oxidative stress and nervous system defects) are able to trigger melanocyte death which frequently embrace the form of apoptosis. 
Melanocytic antigens are able to trigger an immune response by activating the innate immune system and the adaptive immune system, leading to an increased production of auto-antibodies. The high level of melanocyte sensitivity to immune-mediated or toxic-mediated attack compared to fibroblasts and keratinocytes and reduction in the number and dysfunction of keratinocytes with their inability to produce melanocyte growth factors, slowing down the melanocyte development, proliferation and differentiation phenomena are steps to melanocytes disfunction and their consequent destruction.

The mechanisms of cell death at the skin level may be the turntable for complex manifestations and evolution in vitiligo. From clinical point of

\section{Conflict of interest: none declared}

Financial support: none declared

\section{REFERENCES}

1. Chen J, Li S, Li C. Mechanisms of melanocyte death in vitiligo. Med Res Rev. 2021;41 (2):1138-66.

2. Cabrera R, Recule F, Hojman L, Larrondo J. Follicular vitiligo: dermatoscopic features of a new subtype of vitiligo. An Bras Dermatol. 2019;94 (1):120-1.

3. Saurat J-H. Dermatology and sexually transmitted infections. Sixth Edition ed. Issy-les-Moulineaux: Elsevier Masson; 2017. 1288 p.

4. Bergqvist C, Ezzedine K. Vitiligo: A Review. Dermatology. 2020;236 (6):571-92

5. Mubki T, Rudnicka L, Olszewska M, Shapiro J. Evaluation and diagnosis of the hair loss patient: part I. History and clinical examination. J Am Acad Dermatol. 2014;71 (3):415 e1- e15.

6. Rodrigues M, Ezzedine K, Hamzavi I, Pandya AG, Harris JE, Vitiligo Working G. New discoveries in the pathogenesis and classification of vitiligo. J Am Acad Dermatol. 2017;77 (1):1-13.

7. Saurat J-H, Lipsker D, Thomas L, Borradori L, Lachapelle J-M. Dermatology and Sexually Transmitted Infections Sixth Edition. 2017.

8. Patel S, Rauf A, Khan H, Meher BR, Hassan SSU. A holistic review on the autoimmune disease vitiligo with emphasis on the causal factors. Biomed Pharmacother. 2017;92:501-8.

9. Antonelli A, Ferrari SM, Fallahi P. The role of the Th1 chemokine CXCL10 in vitiligo. Ann Transl Med. 2015;3 (Suppl 1):S16.

10. Lai X, Wichers HJ, Soler-Lopez M, Dijkstra BW. Structure of Human Tyrosinase Related Protein 1 Reveals a Binuclear Zinc Active Site Important for Melanogenesis. Angew Chem Int Ed Engl. 2017;56 (33):9812-5.

11. Delmas V, Larue L. Molecular and cellular basis of depigmentation in vitiligo patients. Exp Dermatol. 2019;28 (6):662-6.

12. Prignano F, Pescitelli L, Becatti M, Di Gennaro P, Fiorillo C, Taddei N, et al. Ultrastructural and functional alterations of mitochondria in perilesional vitiligo skin. J Dermatol Sci. 2009;54 (3):157-67.

13. El-Gayyar MA, Helmy ME, Amer ER, Elsaied MA, Gaballah MA. Antimelanocyte Antibodies: A Possible Role in Patients with Vitiligo. Indian J Dermatol. 2020;65 (1):33-7.

14. Strassner JP, Harris JE. Understanding mechanisms of autoimmunity through translational research in vitiligo. Curr Opin Immunol. 2016;43:81-8.

15. Miniati A, Weng Z, Zhang B, Stratigos AJ, Nicolaidou E, Theoharides TC. Neuro-immuno-endocrine processes in vitiligo pathogenesis. Int J Immunopathol Pharmacol. 2012;25 (1):1-7.

16. Kumar R, Parsad D, Kanwar AJ. Role of apoptosis and melanocytorrhagy: a comparative study of melanocyte adhesion in stable and unstable vitiligo. Br J Dermatol. 2011;164 (1):187-91.

17. Richmond JM, Frisoli ML, Harris JE. Innate immune mechanisms in vitiligo: danger from within. Curr Opin Immunol. 2013;25 (6):676-82.

18. Kumar R, Parsad D. Melanocytorrhagy and apoptosis in vitiligo: connecting jigsaw pieces. Indian J Dermatol Venereol Leprol. 2012;78 (1):19-23

19. Kundu RV, Mhlaba JM, Rangel SM, Le Poole IC. The convergence theory for vitiligo: A reappraisal. Exp Dermatol. 2019;28 (6):647-55

20. Seiberg M. Keratinocyte-melanocyte interactions during melanosome transfer. Pigment Cell Res. 2001;14 (4):236-42. view, melanocytic death has a correspondent that embraces the form of white macules on the skin associated or not with leucotrichia.

Despite recent advances in vitiligo research and a better understanding of disfunction and morphological changes in epidermal cells, the treatment of some forms of vitiligo are still a real challenge for clinicians. In this respect, we believe that elucidating the cell death mechanisms at skin level in vitiligo can be an important step in developing some innovative treatment strategies.

\section{Acknowledgement}

All the authors had equal contributions.
21. Bellei B, Pitisci A, Ottaviani M, Ludovici M, Cota C, Luzi F, et al. Vitiligo: a possible model of degenerative diseases. PLoS One. 2013;8 (3):e59782.

22. Henning SW, Jaishankar D, Barse LW, Dellacecca ER, Lancki N, Webb K, et al. The relationship between stress and vitiligo: Evaluating perceived stress and electronic medical record data. PLoS One. 2020;15 (1):e0227909.

23. van den Boorn JG, Picavet DI, van Swieten PF, van Veen HA, Konijnenberg D, van Veelen PA, et al. Skin-depigmenting agent monobenzone induces potent T-cell autoimmunity toward pigmented cells by tyrosinase haptenation and melanosome autophagy. J Invest Dermatol. 2011;131 (6):1240-51.

24. Lee AY, Kim NH, Choi WI, Youm YH. Less keratinocyte-derived factors related to more keratinocyte apoptosis in depigmented than normally pigmented suction-blistered epidermis may cause passive melanocyte death in vitiligo. J Invest Dermatol. 2005;124 (5):976-83.

25. Guerra L, Dellambra E, Brescia S, Raskovic D. Vitiligo: pathogenetic hypotheses and targets for current therapies. Curr Drug Metab. 2010;11 (5):451-67.

26. Plaza-Rojas L, Guevara-Patino JA. The Role of the NKG2D in Vitiligo. Front Immunol. 2021;12:624131.

27. Hassan AS, Kohil MM, Sayed SSE, Mahmoud SB. Immunohistochemical study of perforin and apoptosis stimulation fragment ligand (FasL)in active vitiligo. Arch Dermatol Res. 2020.

28. Toosi S, Orlow SJ, Manga P. Vitiligo-inducing phenols activate the unfolded protein response in melanocytes resulting in upregulation of IL6 and IL8. J Invest Dermatol. 2012;132 (11):2601-9.

29. Moretti S, Fabbri P, Baroni G, Berti S, Bani D, Berti E, et al. Keratinocyte dysfunction in vitiligo epidermis: cytokine microenvironment and correlation to keratinocyte apoptosis. Histol Histopathol. 2009;24 (7):849-57.

30. Samaka RM, Basha MA, Menesy D. Role of Janus kinase 1 and signal transducer and activator of transcription 3 in vitiligo. Clin Cosmet Investig Dermatol. 2019;12:469-80.

31. Fraczek A, Owczarczyk-Saczonek A, Placek W. The Role of TRM Cells in the Pathogenesis of Vitiligo-A Review of the Current State-Of-The-Art. Int J Mol Sci. 2020;21 (10).

32. Giri PS, Patel S, Begum R, Dwivedi M. Association of FOXP3 and GAGE10 promoter polymorphisms and decreased FOXP3 expression in regulatory T cells with susceptibility to generalized vitiligo in Gujarat population. Gene. 2021;768:145295.

33. Zhang S, Liu S, Yu N, Xiang L. RNA released from necrotic keratinocytes upregulates intercellular adhesion molecule-1 expression in melanocytes. Arch Dermatol Res. 2011;303 (10):771-6.

34. Guevara-Patino JA, Plaza-Rojas L. It Takes Two to Tango. J Invest Dermatol. 2020;140 (6):1131-3.

35. Boehncke WH, Brembilla NC. Autoreactive T-Lymphocytes in Inflammatory Skin Diseases. Front Immunol. 2019;10:1198. 